\begin{tabular}{llll}
\multicolumn{2}{l}{ Abstract S76 Table 1 } & & \\
\hline & & Greenwich (\%) & Nepal (\%) \\
& & $\mathrm{n}=86$ & $\mathrm{n}=34245$ \\
\hline Age & -14 & 1.2 & 2.5 \\
& $15-24$ & 34.9 & 20.5 \\
& $25-34$ & 34.9 & 18.0 \\
& $35-44$ & 10.5 & 12.0 \\
& $45-54$ & 10.5 & 15.5 \\
& $55-64$ & 3.5 & 15.5 \\
& $65+$ & 4.7 & 12.0 \\
Site & Pulmonary Smear +ve & 11.6 & 51.8 \\
& Pulmonary Smear -ve & 47.4 & 24.4 \\
& Extrapulmonary & 41.0 & 23.8 \\
Other & Treatment completion & 98 & 91 \\
& TB/HIV co-infection & 1.1 & 2.4 \\
& MDR & 4.7 & 2.6 \\
\hline
\end{tabular}

\section{S77 PRE-TREATMENT CHEST X-RAY SEVERITY AND ITS RELATION TO BACTERIAL BURDEN IN PULMONARY TUBERCULOSIS}

${ }^{1}$ SE Murthy, ${ }^{2} \mathrm{~F}$ Chatterjee, ${ }^{3} \mathrm{PPJ}$ Phillips, ${ }^{4} \mathrm{SR}$ Murray, ${ }^{1} \mathrm{TD}$ McHugh, ${ }^{5} \mathrm{SH}$ Gillespie. ${ }^{1}$ University College London, London, UK; ${ }^{2}$ University College London Hospital, London, UK; ${ }^{3} \mathrm{MRC}$ ) Clinical Trials Unit/UCL, London, UK; ${ }^{4} T B$ Alliance, New York, USA; ${ }^{5}$ University of St Andrews, St Andrews, UK

\subsection{6/thoraxjnl-2014-206260.83}

Background Chest radiographs are commonly used for the diagnosis of tuberculosis and to assess the extent of disease. A relationship between the extent of disease as determined by smear grade and cavitation has been shown to predict 2-month smear results but little has been done to determine whether radiological severity reflects the bacterial burden at diagnosis.

Design/methods Pre-treatment chest X-rays from 1837 subjects with smear positive pulmonary tuberculosis enrolled into the REMoxTB trial were reviewed retrospectively. Two clinicians blinded to clinical details using the Ralph et al scoring system ${ }^{1}$ (comprised of the percentage of affected lung field and presence of cavitation) performed separate readings. An independent reader (a radiologist) reviewed discrepant results for quality assessment and cavity presence. The final cavity assessment was combined with the average percentage affected value to give a severity score (maximum score $100 \%+40$ if any cavitation). The Ralph score was compared to the time to positivity (TTP) of liquid cultures as measured by MGIT 960. The association between CXR severity score and time to positivity (log scale) was evaluated using Spearman's rank correlation coefficient. A Welch t-test was used to compare TTP of those with high and low disease extent and those with and without cavitation.

Results Matching sets of data were available for 1422 subjects. The median severity score was 53.75/140 (IQR 32.03-66.25) and median time to culture positivity $117 \mathrm{~h}$ (4.88 days). CXR severity score was weakly correlated with time to positivity (Spearman's correlation $-0.20, \mathrm{p}<0.0001$ ). Time to positivity was higher in those without cavitation (difference $23.7 \mathrm{~h}$, $\mathrm{p}<0.0001$ ) and those with a low area affected (difference 12.1 $\mathrm{h}, \mathrm{p}<0.0001)$.

Conclusions The radiological severity of pulmonary tuberculosis at diagnosis is weakly correlated with bacterial load as measured by TTP. This suggests that, in addition to bacterial burden, other factors such as immune response influence radiological appearances.

\section{REFERENCES}

1 Ralph AP, Ardian M, Wiguna A, et al. A simple, valid, numerical score for grading chest $X$-ray severity in adult smear-positive pulmonary tuberculosis. Thorax. 2010;65:863-9

\section{S78 DO TUBERCULOSIS CASES MANAGED BY CLINICIANS WITH AVERAGE ANNUAL CASELOADS BELOW 10 HAVE POORER TREATMENT OUTCOMES?}

${ }^{1}$ HR Stagg, ${ }^{2} \mathrm{HL}$ Thomas, ${ }^{2} \mathrm{D}$ Pedrazzoli, ${ }^{2} \mathrm{LF}$ Anderson, ${ }^{1} \mathrm{I}$ Abubakar, ${ }^{3} \mathrm{CS}$ Merle. ${ }^{1}$ University College London, London, UK; ${ }^{2}$ Public Health England, London, UK; ${ }^{3}$ London School of Hygiene and Tropical Medicine, London, UK

10.1136/thoraxjnl-2014-206260.84

Introduction and objectives The 2007 Department of Health Tuberculosis Toolkit advises that clinicians should not be solely managing tuberculosis (TB) cases if their average caseload is less than 10 per year. A systematic evaluation of whether these guidelines are being followed, and how effective such a threshold is, has not been undertaken in the UK.

Methods All UK TB cases notified 2003-2011 were extracted from Public Health England's Enhanced Tuberculosis Surveillance system. Mean caseload for each clinician was calculated over the preceding year and three years by using case notification date. 12 month TB treatment outcomes were categorised as unfavourable or good/neutral. ${ }^{1,2}$ Cases without clinician information and resistant to rifampicin were excluded, the latter due to UK recommendations on the length of treatment. The proportion of cases managed by clinicians with a caseload under 10 was analysed, then random effects logistic regression utilised to determine the relationship between caseload and treatment outcomes, adjusting for clustering by clinician and confounding.

Results 74,550 TB cases were notified 2003-11. The proportion of TB cases seen by a clinician who had a low caseload (less than 10) in the preceding year declined gradually 2004-11 (42 to $28 \%$ ), with no apparent acceleration post-Toolkit. Univariate modelling demonstrated very strong evidence of increased odds of an unfavourable treatment outcome among cases seen by a clinician who had a low caseload over the preceding three years (cluster-specific odds ratio 1.23 (95\% confidence interval 1.141.33 ), p-value $<0.001$ ); this relationship was upheld in a model adjusted for demographic, temporal and clinical confounders (1.14 (1.05-1.23), <0.001; 44,184 cases), and additionally when a sensitivity analysis was performed looking at second assigned clinician, if present.

Conclusions Our analysis indicates that TB cases managed by clinicians with a mean caseload of under 10 over the preceding three years have increased odds of an unfavourable treatment outcome. However other factors, such as the number of clinicians managing TB cases in the hospital overall, should potentially be taken into account for policy recommendations.

\section{REFERENCES}

1 Ditah, Thorax 2008;63:440-446

2 Anderson, Euro Surveill. 2013;18(40):pii=20601

\section{S79 COUGH PREVALENCE AND FREQUENCY IN PULMONARY} TUBERCULOSIS

${ }^{1}$ RD Turner, ${ }^{1} \mathrm{AC}$ Repossi, ${ }^{2} \mathrm{~S}$ Matos, ${ }^{3} \mathrm{SS}$ Birring, ${ }^{1} \mathrm{GH}$ Bothamley. ${ }^{1}$ Homerton University Hospital NHS Foundaiton Trust, London, UK; ' ${ }^{2}$ IEETA, University of Aveiro, Aveiro, Portugal; ${ }^{3}$ King's College London, London, UK

10.1136/thoraxjnl-2014-206260.85 